\title{
Eine Gesundheit
}

Korrespondenzadresse

Prof. Dr. Dr. Manfred Spitzer

Universität Ulm

Abteilung für Psychiatrie

Leimgrubenweg 12-14

87054 Ulm, Deutschland
Bibliografie

DOI https://doi.org/10.1055/a-1193-8611

Nervenheilkunde 2020; 39: 780-790

(C) 2020. Thieme. All rights reserved.

Georg Thieme Verlag KG, Rüdigerstraße 14,

70469 Stuttgart, Germany

ISSN 0722-1541
One Health („eine Gesundheit“) ist eine von der Vereinigung amerikanischer Tierärzte (American Veterinary Medical Association, AVMA) im Jahr 2008 ins Leben gerufene Initiative [32], deren Ziel es ist, die Gesundheit der Menschen, der Tiere und des Planeten Erde multidisziplinär und mit einem alle 3 Bereiche zugleich umfassenden Blick zu betrachten. Das ist beispielsweise bei Krankheiten sehr sinnvoll, die zwischen Tieren und Menschen übertragen werden - man nennt sie seit Mitte des vorletzten Jahrhunderts mit Rudolf Virchow Zoonosen. Wenn man zusätzlich zu den Infektionswegen, der Biochemie und den unterschiedlichen Lebensphasen vieler Krankheitserreger noch deren Lebensräume (irgendwo auf der Welt) und unseren Lebensraum (überall auf der Erde) mit in Betracht zieht, dann folgt der Grundgedanke von One Health fast schon mit zwingender Logik: Mensch, Tierwelt und Lebensraum Erde hängen zusammen.

Daher ist dieser Grundgedanke auch nicht auf die USA beschränkt geblieben. Vielmehr haben sich die Weltgesundheitsorganisation (WHO), die Ernährungs- und Landwirtschaftsorganisation der Vereinten Nationen (Food and Agriculture Organization, FAO) und die Weltorganisation für Tiergesundheit (World Organisation for Animal Health, OIE) diesen Gedanken zu eigen gemacht ( Tab. 1). Auch die US-amerikanischen Centers for Disease Control and Prevention (CDC) und das Deutsche Robert-Koch-Institut (RKI) haben One Health jeweils zu einem ihrer Leitthemen gemacht ( A Abb. 1, \Abb. 2). Die zwischenstaatliche Plattform für Biodiversität und Ökosystem-Dienstleistungen (IMBES) geht nicht zuletzt auf die Gründer von One Health und deren Idee zurück.

Aufgrund der Ausweitung des globalen Reiseverkehrs [9, 12, 39] und Handels [10, 20] wird die Gesundheit zu einem Problem, das sich ebenfalls nur global lösen lässt. Daher sind Zusammenarbeit, Koordination, Kommunikation und konzertierte Aktionen zwischen unterschiedlichen Ländern und Sektoren des Gesundheitswesens, der Veterinärmedizin und der Ökologie unbedingt erforderlich - aber schwierig. Man spricht mittlerweile von der „Schnittstelle Mensch-Tier-Umwelt“ [11], wie beispielhaft anhand dreier Problemkreise leicht zu zeigen ist:

\section{Zoonosen}

Die Mehrheit (etwa 70\%) der neu auftretenden Krankheiten (wie Influenza, HIV/AIDS, Ebola, Zika, Nipah Enzephalitis und zuletzt
Covid-19) sind Zoonosen, d. h. durch Tiere übertragene Krankheiten, die deswegen auch keine politischen Grenzen und definitionsgemäß auch keine biologischen Grenzen zwischen Mensch und Tier kennen. Sie wirken sich mitunter nicht nur auf unsere Gesundheit aus, sondern auch auf die Gesundheit und das Wohlergehen von Tieren und damit auch auf die Landwirtschaft und können zu einer Bedrohung der Nahrungsmittelproduktion führen. So wundert es nicht, dass die erste wesentliche Aktion der genannten, den One-Health-Gedanken vertretenden Organisation in der Erstellung eines Leitfadens zur Bekämpfung von Zoonosen bestand [11].

\section{Antibiotikaresistenz}

Auch Bakterien kennen weder politische noch biologische Grenzen. In der Landwirtschaft bei der Tiermast verwendete Antibiotika haben „Nebenwirkungen“ in Krankenhäusern, denn die gleichen Bakterien, die innerhalb von Tieren eine Resistenz gegenüber Antibiotika entwickeln können, verursachen auch Infektionskrankheiten beim Menschen, deren Behandlung zunehmend schwierig wird, weil die verwendeten Medikamente (Antibiotika) nicht mehr wirken.

\section{Lebensraum und Artenvielfalt}

Die Bedeutung des One-Health-Gedankens wurde nicht zuletzt aufgrund der Corona-Pandemie für viele überhaupt erst erkennbar [6] ${ }^{1}$.Menschen sind nun einmal nicht die einzigen Lebewesen auf der Erde. Insbesondere seit dem Übergang vom Jäger und Sammler zu Ackerbau und Viehzucht leben Mensch mit vielen Arten - Haustieren, Nutztieren, aber auch Parasiten und Schädlingen - eng zusammen. Dies hat in den vergangenen 10000 Jahren zu vielen Epidemien geführt: Die Masern und Tuberkulose verdanken wir den Kühen, den Keuchhusten den Schweinen und die Grippe den Enten. Viele Menschen sind daran gestorben, aber die „Menschheit“ wurde immun -zumindest teilweise, nämlich dort, wo man Haustiere hatte sowie Viehzucht und Milchwirtschaft betrieb. Hunde,

1 Dies gilt auch für den Autor. Bis vor wenigen Wochen hatte ich noch nie davon gehört, musste jedoch im Rahmen meiner - nahezu täglichen - klinischen und wissenschaftlichen Auseinandersetzung mit der Corona-Pandemie unvermeidlich darüber stolpern. 
- Tab. 1 Wichtige Organisationen und Einrichtungen für den One-Health-Gedanken, der auf den Homepages der Institutionen prominent vertreten wird.

\begin{tabular}{|c|c|c|c|c|}
\hline Wer & Gründung & Sitz & $\begin{array}{l}\text { Mitglieds- } \\
\text { staaten }\end{array}$ & Besonderes \\
\hline WHO & 7.4.1948 & Genf & 194 & $\begin{array}{l}\text { Weltgesundheitsorganisation, (WHO, World Health Organization), Organ der } \\
\text { vereinten Nationen (UN) für weltweite Gesundheit. }\end{array}$ \\
\hline FAO & 16.10.1945 & Rom & 194 & $\begin{array}{l}\text { Weltweite Ernährungs- und Landwirtschaftsorganisation (Food and Agricul- } \\
\text { ture Organization) der UN, im deutschen Sprachraum als Welternährungs- } \\
\text { organisation bezeichnet. }\end{array}$ \\
\hline OIE & 25.1 .1924 & Paris & 182 & $\begin{array}{l}\text { Der ursprüngliche Name war Office International des Epizooties (französisch), } \\
2003 \text { umbenannt in World Organization for Animal Health; das alte Akronym } \\
\text { und der Sitz in Paris wurden beibehalten. }\end{array}$ \\
\hline CDC & 1.7.1946 & Atlanta $^{1}$ & USA & $\begin{array}{l}\text { Centers for Disease Control and Prevention, Gesundheitsbehörde der } \\
\text { USA, ursprünglich als Office of National Defense Malaria Control Activities } \\
\text { gegründet, umbenannt am 27.10.1992. }\end{array}$ \\
\hline RKI & 1.7.1891 & Berlin & Deutschland & $\begin{array}{l}\text { Das Robert-Koch-Institut wurde als Königlich Preußisches Institut für } \\
\text { Infektionskrankheiten gegründet und die ersten } 13 \text { Jahre von Robert Koch } \\
\text { geleitet. Im Jahr } 1912 \text { erhielt es (zum 30. Jahrestag der Entdeckung des } \\
\text { Tuberkel-Bazillus) den Namenszusatz „Robert Koch“, nach dem Ersten Welt- } \\
\text { krieg verschwand „Königlich“ aus dem Namen und im Jahr } 1942 \text { als selbst- } \\
\text { ständige „Reichsanstalt“ den heutigen Namen. }\end{array}$ \\
\hline IPBES & 21.4.2012 & Bonn & 136 & $\begin{array}{l}\text { Intergovernmental Science-Policy Platform on Biodiversity and Ecosystem } \\
\text { Services (IPBES), zu Deutsch: Zwischenstaatliche Plattform für Biodiversität } \\
\text { und Ökosystem-Dienstleistungen, auch Weltbiodiversitätsrat oder Weltrat } \\
\text { für Biologische Vielfalt genannt. Die Organisation unter dem Dach der UN } \\
\text { hat } 136 \text { Mitgliedsstaaten. Ihre Aufgabe ist die wissenschaftliche Politikbera- } \\
\text { tung in Sachen Nachhaltigkeit, biologische Vielfalt und „Ökosystemdienst- } \\
\text { leistungen“. }\end{array}$ \\
\hline IUCN & 5.10 .1948 & Gland & 208 & $\begin{array}{l}\text { International Union for Conservation of Nature (IUCN), zu deutsch: Interna- } \\
\text { tionale Union zur Bewahrung der Natur. Die IUCN ist eine zwischenstaatliche } \\
\text { Organisation, die sich sowohl aus staatlichen als auch zivilgesellschaftlichen } \\
\text { Mitgliedsorganisationen zusammensetzt. Sie macht sich die Erfahrung, die } \\
\text { Ressourcen und die Reichweite ihrer mehr als } 1400 \text { Mitgliedsorganisationen } \\
\text { und den Input von mehr als } 17000 \text { Experten zunutze. Diese Vielfalt und das } \\
\text { große Fachwissen machen die IUCN zur weltweiten Autorität für den Status } \\
\text { der natürlichen Welt und die zu ihrem Schutz erforderlichen Maßnahmen. }\end{array}$ \\
\hline
\end{tabular}

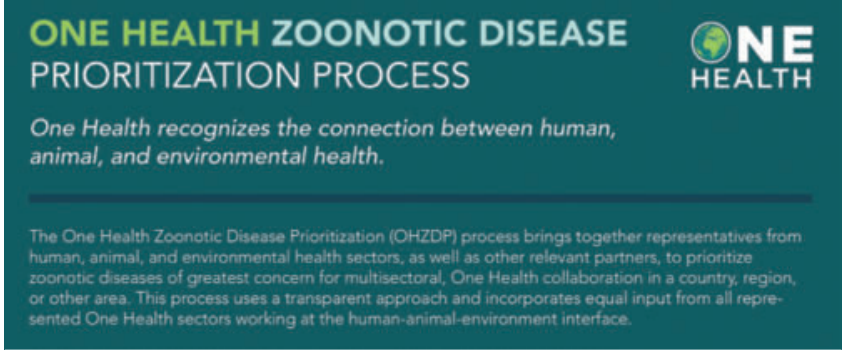

- Abb. 1 Faksimile (Screenshot) eines Ausschnitts der Webseite des CDC zum Thema One Health (https://www.cdc.gov/onehealth/ what-we-do/zoonotic-disease-prioritization/fact-sheet.html; abgerufen am 10.10.2020).

Pferde, Esel, Katzen, Rinder, Schweine, Schafe, Gänse, Hühner und Enten - diese Haus- und Nutztiere trugen die Krankheiten unserer Vorfahren in sich. Für uns heutige Menschen sind dagegen Fledermäuse, Schuppentiere, Schleichkatzen oder Schlangen Überträger von Krankheiten und zwar nicht deswegen, weil diese Tiere uns bedrängen, sondern wir sie. - Durch die immer weiter fortschreiten-

\section{Das One-Health-Konzept}

Mehr als die Hälfte aller bekannten Erreger, die Erkrankungen beim Menschen hervorrufen, sind so genannte Zoonose-Erreger. Diese Bakterien, Pilze, Viren und Parasiten können zwischen Mensch und Tier übertragen werden.

Eine wachsende Bevölkerung, steigende Mobilität, schwindende Lebensräume, industrielle Landwirtschaft und intensivierte Nutztierhaltung - all dies sind Faktoren, die das Risiko für eine schnelle weltweite Ausbreitung von Krankheitserregern erhöhen.

Die Gesundheit von Mensch, Tier und Umwelt sind eng miteinander verknüpft. Beim One-Health-Ansatz arbeiten die Akteure der verschiedenen Disziplinen Humanmedizin, Veterinărmedizin, Umweltwissenschaften - fächerübergreifend zusammen, um beispielsweise der Öbertragung von Krankheitserregern entgegenzuwirken. Auch im Kampf gegen Antibiotikaresistenzen ist der OneHealth-Ansatz zentral: Resistenzen kennen keine Grenzen und können sich zwischen Mensch, Tier und Umwelt rasch verbreiten.

Stand: 09.05.2019

Abb. 2 Faksimile (Screenshot) eines Ausschnitts der Webseite des RKI zum Thema One Health (https://www.rki.de/DE/Content/ Infekt/Antibiotikaresistenz/One-Health/One_Health-Konzept.html; abgerufen am 10.10.2020) 


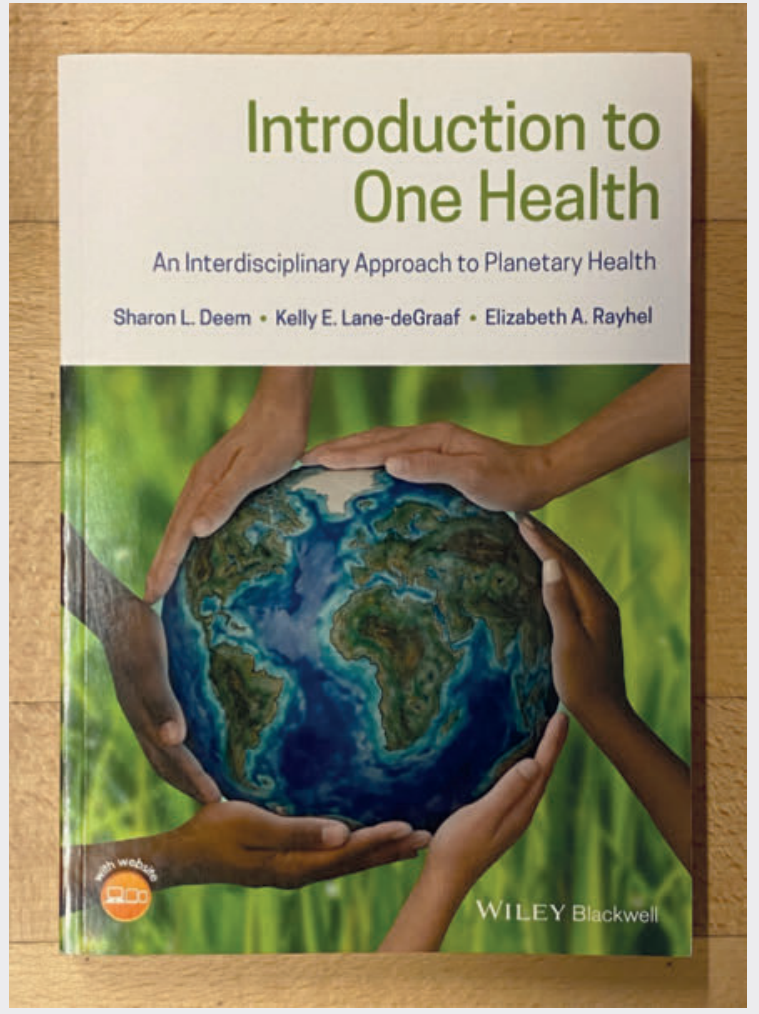

- Abb. 3 Cover des Einführungsbuchs zum One-Health-Gedanken.

de Abholzung der Wälder und die zunehmende Urbanisierung vieler Regionen engen wir den Lebensraum vieler Tiere ein, wodurch ihre Nähe zu uns und damit auch die Wahrscheinlichkeit zunimmt, mit ihnen in Kontakt kommen, sodass Krankheitserreger (Malaria, Ebola, Corona) von ihnen auf uns überspringen können.

Die WHO, FAO und OIE haben daher im Jahr 2019 gemeinsam einen „Dreigliedrigen Leitfaden für Zoonosen“ (Tripartite Zoonoses Guide, TZG) herausgegeben, um die Länder der Vereinten Nationen in ihren Bemühungen um die Gesundheit von Mensch, Tier und Erdball zu unterstützen. Er bezieht alle relevanten Sektoren, Fachkenntnisse, Perspektiven und Erfahrungen ein und vernetzt nationale Gruppen, sodass koordinierte internationale Reaktionen auf globale Bedrohungen möglich werden. Einige heben hervor, dass zu diesem Wissen auch die Erfahrungen indigener Völker mit dem von ihnen bewohnten Land (immerhin etwa ein Viertel der Erde) und der von ihnen oft betriebenen schonenden, konservierenden Nutzung gehören [27, 31]. Schon im Jahr 2018 erschien eine von Wissenschaftlern der University of Missouri in Columbia, Missouri sowie der der Fontbonne University in St. Louis, Missouri, herausgegebene Einführung in One Health ( $\triangleright$ Abb. $\mathbf{3}$ ), die dessen Grundgedanken und einige Beispiele hierzu in 15 Kapiteln durchdekliniert [16].

\section{Nahrungsmittelknappheit}

So wird beispielsweise die weltweite Nahrungsmittelknappheit diskutiert [16]. Wussten Sie, dass die globalen Vorräte an Weizen für etwa 60 Tage ausreichen, und dass die Weizenpreise deutlich stei-

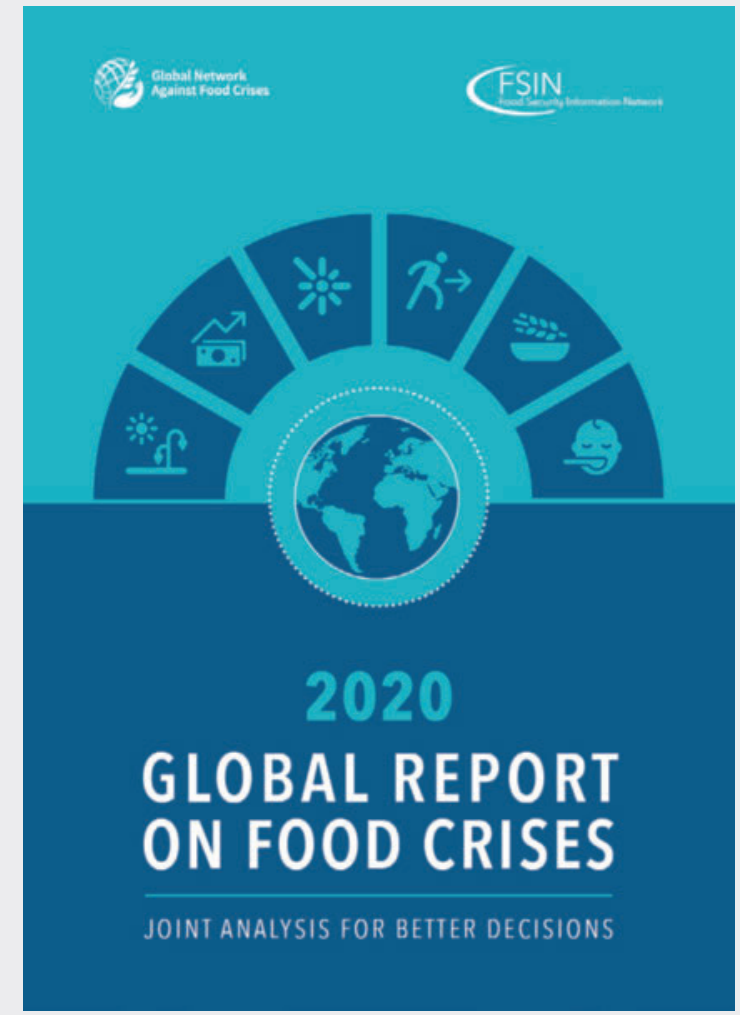

- Abb. 4 Der neueste Report des Netzwerks für Informationen zur Sicherheit der Nahrungsmittelversorgung (Food Security Information Network, FSIN 2020) befasst sich mit den Auslösern für Hungersnöte. Dargestellt auch als Icons auf dem Titelblatt des Reports.

gen, wenn es ein paar Tage weniger werden? Weil Transport sehr günstig und irgendwo auf der Welt immer gerade Ernte ist, unterliegen die Preise für Weizen - und für viele Nahrungsmittel ganz allgemein - heute deutlich geringeren Schwankungen als vor 50 , 100 oder 300 Jahren [34]. Das System hat aber seine Schwächen: Während der Finanz- und Wirtschaftskrise 2008/2009 kam es beispielsweise zu deutlich gesteigerten Nahrungsmittelpreisen. Dies macht in „reichen“ Ländern kaum Probleme, denn wer $10 \%$ seines Einkommens für Nahrungsmittel ausgibt, den trifft eine Steigerung der Preise um 50 \% kaum, kann er doch die zusätzlichen benötigten $5 \%$ seines Einkommens leicht anderswo einsparen. In einem ärmeren Land hingegen, dessen Einwohner im Mittel $80 \%$ ihres Einkommens für Nahrungsmittel ausgeben, führt eine Preissteigerung um $50 \%$ dazu, dass selbst im besten Fall (wenn man das ganze Einkommen für Nahrungsmittel ausgibt) nur 100 von benötigten $130 \%$ des benötigten Einkommens zur Verfügung steht, also gehungert werden muss. Hunger wiederum führt zu Unruhen, vor allem dann, wenn das Durchschnittsalter der Bevölkerung noch niedrig ist - wie man während der Finanzkrise vor gut 11 Jahren weltweit in mehr als 20 Ländern gesehen hat [36, 38, 43]. Auch die gegenwärtige Corona-Krise kann zu Problemen in der Logistik und damit der Verteilung von Nahrungsmitteln beitragen, selbst wenn es global betrachtet prinzipiell an nichts fehlt, worauf Wissenschaftler der Plattform Globales Netzwerk Gegen Nahrungsmittelkrisen schon vor Monaten hinwiesen [17] ( $\triangleright$ Abb. 4). 


\section{Lebensraum}

Vor 300 Jahren, also im Jahr 1700 wurden lediglich 9\% der Landerdoberfläche für den Ackerbau verwendet. Im Jahr 1992 waren es $40 \%$ [28]. Immer mehr Land wird zur Nahrungsmittelproduktion urbar gemacht, weswegen es weniger unberührte Natur und weniger Lebensraum für sehr viele Tierarten gibt. Menschen kommen den Tieren immer näher, was die Verbreitung von Zoonosen begünstigt. Als Beispiel für die nahezu beliebig hohe Komplexität des Geschehens sei Folgendes angeführt: Eine internationale Arbeitsgruppe (auch unter deutscher Beteiligung u. a. mit dem Virologen Christian Drosten) publizierte im April 2020 eine Studie zu den Wechselwirkungen zwischen Mensch und Tier mit Blick auf die Zusammenhänge zwischen Stress und Immunsystem. Die Höhle von Faucon im zentralafrikanischen Gabun, einer ehemaligen französischen Kolonie, ist die größte von allen untersuchten Höhlen und beherbergt eine Vielfalt von Fledermausarten. Sie wird regelmäßig von Dorfbewohnern besucht, um Fledermäuse für den Verzehr zu jagen. Man geht davon aus, dass solche Störungen des Lebensraums bei den Fledermäusen chronischen Stress bewirken und damit bei den Tieren eine Unterdrückung der körpereigenen Abwehr (Immunsuppression) verursachen. Hierdurch wiederum könnte die Anfälligkeit der Tiere für Infektionen mit Viren zunehmen [29]. Durch den häufigen Kontakt kann es dann zur Übertragung auf den Menschen kommen, wie dies für Coronaviren mittlerweile 2-mal geschehen ist. Eine Fledermaus frisst täglich 350 Insekten, die wiederum Malaria übertragen, weswegen die Bedrohung der Fledermäuse nicht nur für die Verbreitung von Covid-19, sondern auch von Malaria relevant ist.

\section{Artensterben}

Die Zahl der Tier- und Pflanzenarten wurde vor etwa 25 Jahren durch das Global Biodiversity Assessment im Auftrag der UN mit rund 1,75 Millionen ermittelt [40]. Diese Zahl ist nur ein Schätzwert. Wissenschaftler schätzen, dass es zwischen 10 und 100 Millionen Tier- und Pflanzenarten auf der Erde gibt. Der Artenreichtum nimmt jedoch mit zunehmender Geschwindigkeit seit Jahrzehnten ab, was meistens als abnehmende Biodiversität bezeichnet wird, obgleich dieser Begriff auch die abnehmende genetische Verschiedenheit (der einzelnen Individuen innerhalb einer Art) und die abnehmende Verschiedenheit von Lebensräumen meinen kann. Man schätzt, ${ }^{2}$ dass etwa $40 \%$ der 310000 Pflanzenarten, die es weltweit gibt [16], vom Aussterben bedroht sind ( $\bullet$ Abb. 5). War noch im Jahr 2016 im ersten Report über den Zustand der Pflanzenwelt von $20 \%$ bedrohter Arten die Rede, so zeigten genauere Daten in den 4 Jahren danach eine doppelt so hohe Zahl [5].

Die Hauptursache der Bedrohung vieler Pflanzenarten vom Aussterben ist nicht die Erderwärmung (obgleich deren Bedeutung zunimmt, liegt ihr Anteil derzeit nur bei etwa $4 \%$ ), sondern die Konversion von immer mehr unberührter Natur in Ackerbauflächen (mit einem Anteil von 38\% am Gesamtgeschehen): „Noch nie zuvor war die Biosphäre, diese dünne Schicht des Lebens, die wir unsere Heimat nennen, so intensiv und dringend bedroht wie heute. Die Rate der Rodung von Wäldern ist stark gestiegen, damit wir genü-

2 Es wurden erst etwa 15\% aller Pflanzenarten daraufhin untersucht [26].

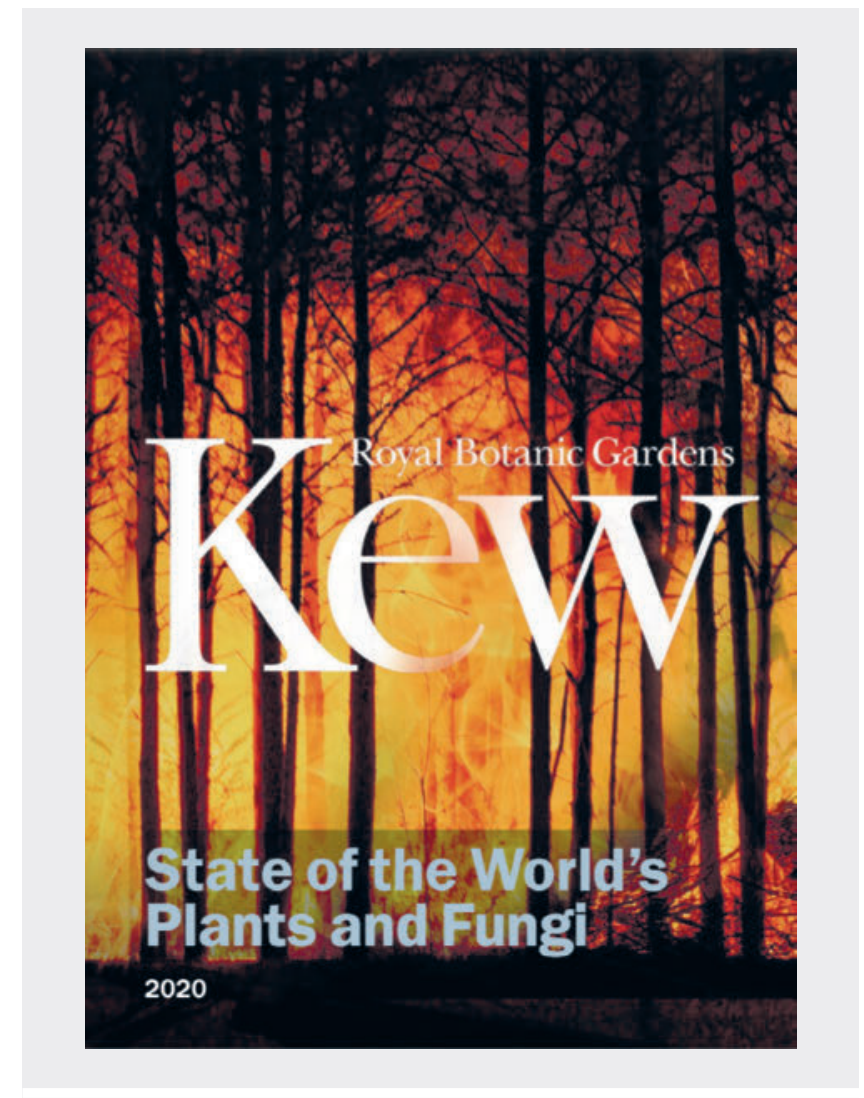

- Abb. 5 Im neuesten Berichts einer Arbeitsgruppe an den Royal Botanic Gardens (im Londoner Stadtteil Kew) über den Zustand des Pflanzenreichs [5]. Nach Berichten über den Zustand der Pflanzen aus den Jahren 2016 und 2017 sowie über den Zustand der Pilze (2018) ist es der vierte seiner Art, der auf 100 Seiten eine sehr gründliche Darstellung und Analyse der vorhandenen Erkenntnisse liefert. Einer der Schwerpunkte ist, wie auch auf dem Titel thematisiert, die weltweit wachsende Anzahl von Waldbränden.

gend Land haben, um immer mehr Menschen zu ernähren“, 3 beschreiben Antonelli und seine Mitarbeiter die Situation [5]. Unter den vom Aussterben bedrohten Pflanzenarten sind übrigens auch 723 in der Medizin als Heilmittel eingesetzte Arten [5].

Im Tierreich besonders vom Aussterben bedroht sind die Amphibien (Frösche, Salamander, Lurche): Etwa ein Drittel der 6300 Arten ist bedroht [45]. Dies liegt vor allem an der Zerstörung ihrer Lebensräume. Durch Ausrottung sind dagegen viele größere Land- und Meerestiere ausgestorben oder vom Aussterben bedroht. Hinzu kommt als dritter Faktor der weltweite Verkehr und damit die Zunahme der Konkurrenz unter Arten, die früher abgegrenzt voneinander existiert hatten. Schließlich macht der Klimawandel vielen Arten zu schaffen. Eine im Fachblatt Science publizierte Analyse von 131 Studien zu den Ursachen des Artensterbens im Tierreich ergab, dass etwa $8 \%$ vom Klimawandel verursacht werden [42]. Dieser Effekt hat - wie der Klimawandel selbst - eine steigende Tendenz und wurde mittlerweile experimenzell in einer Arbeit mit dem bezeichnenden Titel „Lebe schnell und stirb jung: Experimenteller Nach-

3 "Never before has the biosphere, the thin layer of life we call home, been under such intensive and urgent threat. Deforestation rates have soared as we have cleared land to feed ever-more people." 
weis der Auswirkungen des Klimawandels auf das Aussterben von Populationen"4 nachgewiesen [8].

Im Hinblick auf die Tierwelt - etwa 1400000 wirbellose Tierarten und 62000 Arten von Wirbeltieren - sieht es also nicht besser aus als bei den Pflanzen. Man spricht mittlerweile erdgeschichtlich von der sechsten großen Welle des Aussterbens von Arten, wobei die erste vor über einer Milliarde Jahren im Aussterben der Lebewesen durch Vergiftung mit Sauerstoff (den die Pflanzen produzierten) bestand und die fünfte große Welle vor 66 Millionen Jahren (bei dem u. a. die Dinosaurier ausstarben) höchstwahrscheinlich auf einen Meteoriteneinschlag im Golf von Mexiko zurückgeht [21, 22, 32], nachdem dieser Gedanke im Jahr 1980 erstmals publiziert wurde [4]. Weil man damals aber den weltgrößten Meteoritenkrater noch nicht kannte, wurde diese Idee jedoch zunächst sehr skeptisch betrachtet, ist aber mittlerweile weitgehend akzeptiert.

Innerhalb weniger Jahrzehnte nahmen nicht nur die Populationen von Insekten, Vögeln, Fischen und Säugetieren deutlich ab, auch die Vielfalt der Arten ist bedroht. Seit 1964 führt die Weltnaturschutzunion (IUCN) die „internationale Rote Liste gefährdeter Tier- und Pflanzenarten“. Sie unterscheidet die unterschiedlichen Gefährdungsstufen, von „nicht gefährdet“ über „verletzlich“, „stark gefährdet“ oder „vom Aussterben bedroht“ bis „ausgestorben“. Es sind noch längst nicht alle gefährdeten Arten einer Bewertung unterzogen, aber immerhin wurde schon der Gefährdungsstatus aller Vögel, Säugetiere und Amphibien beurteilt. Im Jahr 2020 galten 32441 der mehr als 120000 untersuchten Tier- und Pflanzenarten als vom Aussterben bedroht, im Jahr 2000 waren dies noch 11046 von 16507 und im Jahr 2010 bereits 18351 von 55926 untersuchten Arten.

\section{Anthropozän: Ein neuer Name für die Bedrohung der Welt durch den Menschen}

Demgegenüber war man sich von Anfang an darüber einig, dass die seit einigen Jahrzehnten oder vielleicht schon seit mehr als 2 Jahrhunderten ${ }^{5}$ (erdgeschichtlich macht das kaum einen Unterschied) sechste große Welle des Artensterbens nicht auf Geophysik, Vulkanismus oder Meteoriten zurück geht, sondern auf die Aktivitäten des Menschen. Nicht zuletzt nennt man deswegen auch das derzeitige Zeitalter der Erdgeschichte das Anthropozän. Das Wort „Anthropozän“ wird (noch inoffiziell) zur Benennung einer neuen geochronologischen Epoche verwendet, in der wir begonnen haben zu leben: Der Name soll andeuten, dass der Mensch zu einem der wichtigsten Einflussfaktoren auf die biologischen, geologischen und atmosphärischen Prozesse auf der Erde geworden ist. Der Begriff wurde Anfang dieses Jahrhunderts vom niederländischen Meteorologen und Nobelpreisträger für Chemie, Paul J. Crutzen, wieder in die Diskussion eingebracht $[14,15,35]$, nachdem der italienische Geologen Antonio Stoppani bereits im Jahr 1873 die Bezeichnungen „Anthropozoischen Ära“ beziehungsweise „Anthropozoi-

4 "Live Fast, Die Young: Experimental Evidence of Population Extinction Risk due to Climate Change."

5 Der Chemie-Nobelpreisträger Paul Cruzen schrieb im Jahr 2002 in Nature: „The Anthropocene could be said to have started in the late eighteenth century, when analyses of air trapped in polar ice showed the beginning of growing global concentrations of carbon dioxide and methane." kum“ als Bezeichnungen für ein neues Erdzeitalter vorgeschlagen hatte ( $\triangleright$ Abb. 6).

\section{Verlust der Biodiversität und Corona-Krise}

Die früheren Wellen des Aussterbens und deren Ursachen werden anhand von Fossilien und anderen erdgeschichtlichen Datenquellen (Bohrungen in Gestein und Eis) zu rekonstruieren versucht. Das jetzige Aussterben im Bereich der Tierwelt wird dagegen dokumentiert. Was mit einem Treffen unter der Schirmherrschaft der National Academy of Science und des Smithsonian Institut in Washington, DC, unter dem Titel „National Forum on BioDiversity“6 im September 1986 begann, wurde innerhalb kurzer Zeit zu einem bedeutsamen wissenschaftlichen und mittlerweile auch politischen Begriff. Das ökologische Gleichgewicht ist recht empfindlich. Stirbt beispielsweise eine Pflanzenart aus, von der sich eine bestimmte Insektenart vorwiegend ernährt, ist auch diese in ihrem Bestand gefährdet. Davon können wiederum Wirbeltiere (Vögel, Fledermäuse) abhängen, die sich von Insekten ernähren. An fast allen Ursachenverkettungen für das Aussterben von Arten ist der Mensch beteiligt: intensive Landwirtschaft mit vorwiegend Monokulturen, Insektizide, Pestizide,

6 Ursprünglich wurde „Biodiversity“ als Kürzel für „biological diversity“ gebraucht, wie man im Titel der Tagung - BioDiversity - noch erkennen kann. 


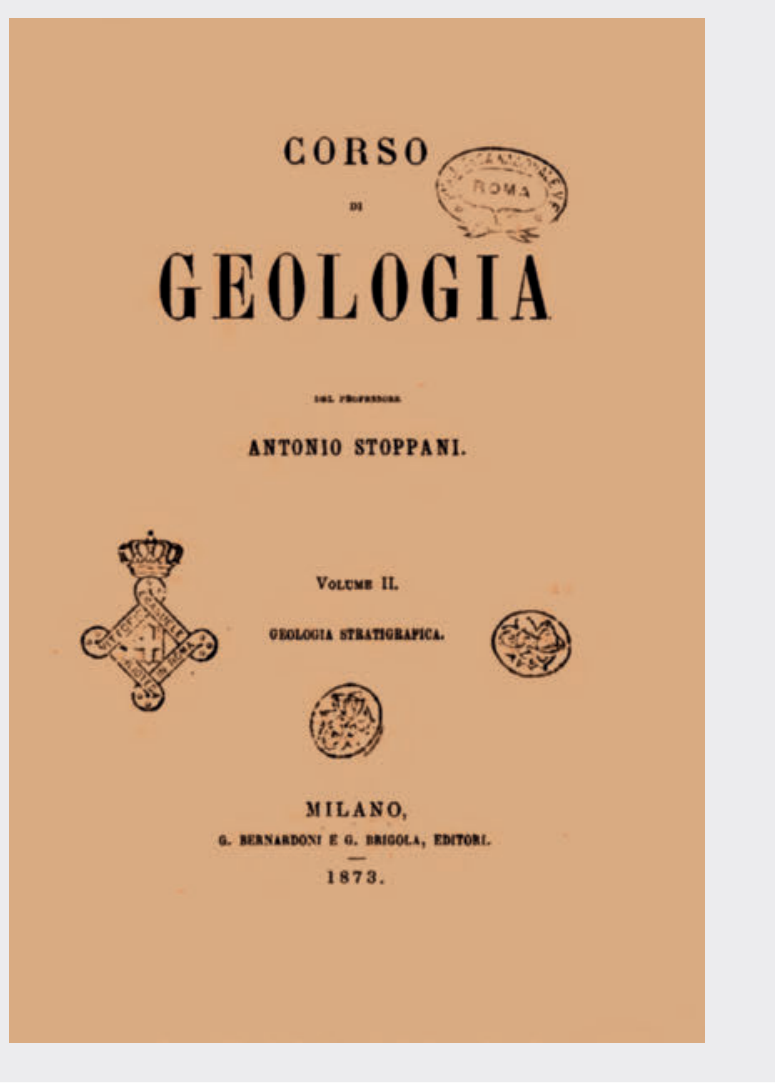

- Abb. 6 Titelseite der Schrift des italienischen Geologen Antonio Stoppani, der dort auf S. 463 bereits im Jahr 1873 von der „antropozoischen Ära" spricht.

vergiftete Lebensräume, Eindringen fremder Arten in Ökosysteme durch den weltweiten Handel etc. Hinzu kommt der Landverbrauch durch Bebauung und Verkehrswege sowie die Tatsache, dass in den Industrieländern immer weniger Obst-, Gemüse- und Getreidesorten kultiviert werden. Beispielsweise handelt es sich bei den gängigen Kartoffel- und Apfelsorten, die überall zu kaufen sind, nur um einen winzigen Bruchteil der tatsächlichen Sorten, wodurch die meisten nach und nach in Vergessenheit geraten und langsam aussterben.

Es ist zunächst gar nicht so einfach, sich zu erklären, warum Biodiversität einen Wert an sich darstellt. „Vielfalt ist immer besser als Einfalt“, sagen die einen. „Monokulturen sind effektiver“ und „Artensterben gehört zur Evolution“, entgegnen die anderen. Mittlerweile wurde empirisch gefunden, dass im Laufe der Erdgeschichte nichts beständiger war als der Wechsel und damit das Aussterben von Arten [1, 2]. Warum muss uns dann die derzeitige Abnahme der Biodiversität Sorgen bereiten? Sie ist zum einen stärker ausgeprägt als je zuvor: Innerhalb weniger Jahrzehnte starben etwa 1000-mal mehr Arten pro Zeiteinheit aus als dies normalerweise der Fall ist. Zum zweiten konnte experimentell nachgewiesen werden, dass eine größere Artenvielfalt ein Ökosystem gegenüber „Ausbeutung“ widerstandsfähiger macht. Mehr Artenvielfalt bewirkt, dass für bestimmte Funktionen im System mehrere Träger vorhanden sind, die diese Funktion innehaben. Bei Ausfall eines Trägers können daher andere einspringen und die Funktion ist noch immer gewährleistet. Das System hat durch Artenvielfalt also eine eingebaute Redundanz, die es unempfindlicher gegenüber Störun- gen von außen macht [3]. In heutigen Ökosystemen haben solche Störungen überall fast immer die gleiche Ursache: Eingriff und Ressourcenverbrauch ${ }^{7}$ durch den Menschen.

Zur besseren Überwachung (Monitoring und Kontrolle) des Artensterbens wurde 2012 - im Wesentlichen von den Gründern von „One Health“ - die Intergovernmental Platform on Biodiversity and Ecosystem Services (IPBES) mit Sitz (Sekretariat) in Bonn von 90 Staaten gegründet. Das zwischenstaatliche Gremium soll helfen, umweltpolitische Entscheidungen nach bestem Stand des Wissens zu treffen. Politische Entscheidungsträger können Anfragen an IPBES stellen, wo dann der aktuelle Stand des Wissens zum jeweiligen Problem in einem sog. Assessment zusammengetragen wird, um Handlungsoptionen und deren Folgen aufzuzeigen [24]. Die neueste, am 29.Oktober 2020 erschienene Publikation von IMBES dreht sich um den Zusammenhang von Zoonosen (und damit auch der CoronaPandemie) und Biodiversität. Dort schreiben 22 Experten aus verschiedenen Ländern: „Pandemien nehmen zwar ihren Ursprung von Erregern, die in tierischen Reservoiren vorkommen, aber entstehen letztlich durch menschliche Aktivitäten. Die den Pandemien zugrunde liegenden Ursachen sind dieselben globalen Umweltveränderungen, die den Verlust der Biodiversität und den Klimawandel vorantreiben. Dazu gehören Veränderungen der Landnutzung, die Ausdehnung und Intensivierung der Landwirtschaft sowie der Handel und Konsum von Wildtieren. Diese Triebkräfte des Wandels bringen Wildtiere, Vieh und Menschen in engeren Kontakt, sodass tierische Mikroben in den Menschen eindringen und zu Infektionen, manchmal zu Ausbrüchen, und seltener zu echten Pandemien führen, die sich über Straßennetze, städtische Zentren und globale Reise- und Handelsrouten ausbreiten "8 [25]. Die Autoren rechnen ferner vor, dass die Drosselung von Landverbrauch und Handel mit Wildtieren als Nahrungsquelle sowie ein globales Überwachungssystem für die Gesundheit von Mensch, Tier und Globus nur einen Bruchteil dessen kosten würden, was die gegenwärtige Pandemie weltwirtschaftlich kostet, volle 2 Größenordnungen weniger, also etwa ein Hundertstel. Anders ausgedrückt: Den One-Health-Gedanken nicht ernst zu nehmen, können wir uns im Grunde gar nicht leisten.

\section{Earth Overshoot Day}

Man kann versuchen, die biologischen „Leistungen“ der Erde und den „ökologischen Fußabdruck der Menschheit“ mit Hilfe von publizierten Daten zur Wirtschaft und zum Verbrauch zu berechnen. Im Jahr 1961 wurde dies erstmals gemacht, wodurch man fand,

7 Ökonomen sprechen im Hinblick auf diesen Eingriff und Ressourcenverbrauch auch von der Nutzung von „Ecosystems Services.“ Dieser Euphemismus klingt deutlich besser als „Ausbeutung der Natur“ - ist jedoch nichts weiter als ein gefälligerer Name.

8 "Pandemics have their origins in diverse microbes carried by animal reservoirs, but their emergence is entirely driven by human activities. The underlying causes of pandemics are the same global environmental changes that drive biodiversity loss and climate change. These include land-use change, agricultural expansion and intensification, and wildlife trade and consumption. These drivers of change bring wildlife, livestock, and people into closer contact, allowing animal microbes to move into people and lead to infections, sometimes outbreaks, and more rarely into true pandemics that spread through road networks, urban centers and global travel and trade routes." 


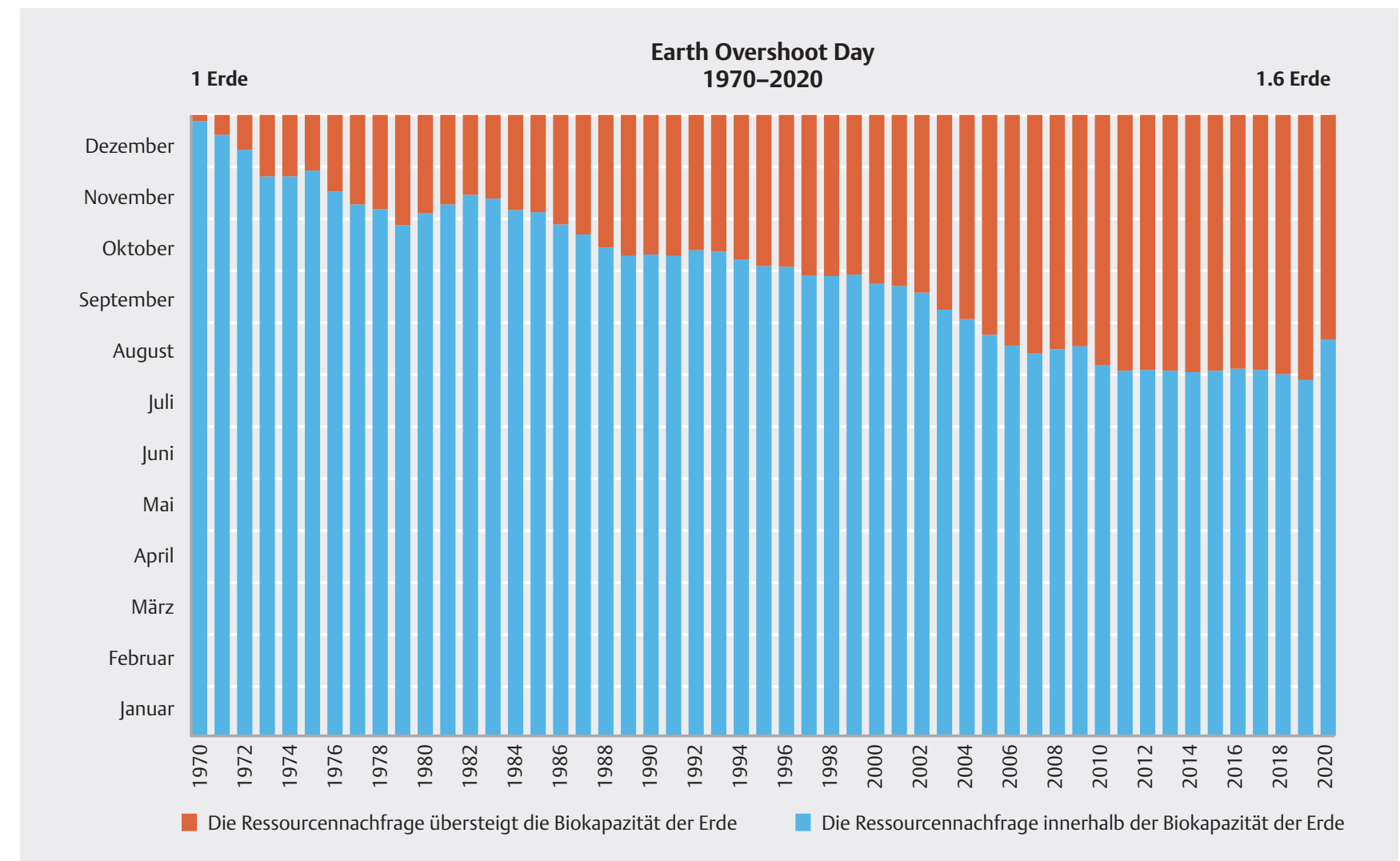

Abb.7 Der Tag, an dem wir die Ressourcen, welche die Erde in einem Jahr bereitstellen kann, schon verbraucht haben, ist innerhalb der letzten 50 Jahre immer früher eingetreten. War dies im Jahr 1970 noch erst am 29.12. der Fall, wurde er für 1980 schon für den 4. November berechnet, für 1990 am 11. Oktober, für das Jahr 2000 am 23. September, 2010 am 7. August, 2019 am 29 Juli und aufgrund des Wirtschaftseinbruchs durch die Corona-Pandemie im Jahr 2020 erst am 7. August und damit zum ersten Mal deutlich später im Jahr, wie man an der blauen Säule ganz rechts sieht. Auch bei der Ölkrise 1973/74 und der Finanzkrise 2008/9 war dies zu verzeichnen (nach Daten aus [18])

dass die Menschheit damals $73 \%$ der Ressourcen verbrauchte, die der Planet Erde bereitstellte. Seit dem Jahr 1970 ist dies grundlegend anders: In diesem Jahr überstieg der Fußabdruck erstmals die Biokapazität der Erde. Seit 1986 wird jährlich der „Earth Overshoot Day" berechnet, indem man die Biokapazität der Erde durch den ökologischen Fußabdruck der Menschheit dividiert und das Ganze mit 365 multipliziert. Dann erhält man den Tag im Jahr, an dem die Ressourcen, die weltweit verbraucht werden, die Kapazität der Erde, diese nachhaltig zu reproduzieren, übersteigt ( $\bullet \mathbf{A b b}$. 7).

Man kann den Quotienten auch umgekehrt bilden (ökologischen Fußabdruck der Menschheit dividiert durch die Biokapazität der Erde). Dieser Wert gibt an, wie viele Erden man bräuchte, um so zu leben, wie wir das gerade tun. Derzeit liegt dieser Wert bei 1,6 . Wir haben jedoch nur eine, woraus sich ergibt, dass wir im Hinblick auf unsere Umwelt jährlich über unsere Verhältnisse leben. Methodenkritisch ist sicherlich einzuwenden, dass die beiden ins Verhältnis gesetzten Größen (Kapazität und Fußabdruck) nicht genau zu messen sind. Sie werden auch immer wieder neu und etwas anders berechnet, wobei man dann nicht nur den neuen Wert berechnet, sondern alle früheren Werte noch einmal, um ihre Vergleichbarkeit zu wahren [18]. Man kann also früher ermittelte Werte nicht mit den heutigen Werten vergleichen, wohl aber die für heute berechneten Werte mit denen für frühere Zeitpunkte berechneten Werten. Man kann den Earth Overshoot Day auch nach Ländern getrennt berechnen. Dann belegten im Jahr 2020 Qatar und Luxemburg die Plätze 1 (16. Februar) und 2 (19. Februar). Für die USA lag der Earth Overshoot Day in 2019 am 15. März, für die EU am 10. Mai und für Deutschland am 3. Mai. Würde die gesamte Weltbevölkerung so leben wie die USA, bräuchten wir 5 Erden. Was bedeutet es dann, wenn der ehemalige US-Präsident George Bush (Senior) im Jahr 1992 schon vor der Weltklimakonferenz in Rio sagte "the American way of life is not negotiable"??

9 Im Jahr 1997 wurde ein verbindliches Abkommen in Kyoto ausgehandelt, aus dem die USA jedoch unter George W. Bush (Junior) 2001 austraten, nachdem der UN-Klimarat (IPCC) zuvor einen Bericht publiziert hatte, dem zufolge die USA für ein Viertel des globalen Ausstoßes der Treibhausgases $\mathrm{CO}_{2}$ verantwortlich sind [7]. Wenige Monate danach wurde das Kyoto-Protokoll ohne die USA auf der Weltklimakonferenz in Bonn angenommen, wonach in den verbleibenden Ländern die Ratifizierung beginnen konnte. Erst 2015 gelang es in Paris, 195 Staaten, einschließlich der USA, auf das Ziel von maximal $1,5^{\circ} \mathrm{C}$ Erderwärmung einzuschwören. Am 1. Juni 2017 verkündete Donald Trump, dass die USA aus dem Klimaschutzabkommen austreten und „sabotiert [...] als Land mit den weltweit zweitgrößten Treibhausgasausstoß die internationalen Klimaschutzbemühungen schon kurz nach dem Inkrafttreten des Pariser Abkommens. Aber zum Glück steht der amerikanische Präsident mit seiner Position allein da. Nicht mal in seinem eigenen Land gehen alle den Kohlekurs des Präsidenten mit. Viele Städte, Gemeinden und Regionen machen trotzdem weiter mit ihren Klimaschutzmaßnahmen“, kann man dazu noch bei Greenpeace [19] nachlesen. 


\section{Röteln}

Die Röteln sind eine hochansteckende, durch Rötelnviren über die Luft verbreitete, weltweit auftretende Infektionskrankheit. Wer einmal infiziert war, ist lebenslang gegenüber dem Rubella-Virus immun, weshalb man die Röteln zu den Kinderkrankheiten zählt. Die klinischen Erscheinungen - masernähnliche rote Hautflecken, Fieber und Lymphknotenschwellungen - wurden von den beiden deutschen Ärzten de Bergan und Orlow zuerst beschrieben, weswegen die Röteln im englischen Sprachgebrauch auch als „Deutsche Masern“ (German measles; im Gegensatz zu den Masern: English measles) bezeichnet werden. Wegen der hohen Infektiosität (der R-Wert der Röteln liegt zwischen 3,5 und 7,8) und weil eine Rötelninfektion während der Schwangerschaft zu schweren Komplikationen (Rötelnembryofetopathie mit z. B. Herzfehler, Katarakt, Innenohrschwerhörigkeit bzw. Mikrozephalie) und zu Fehlgeburten führen kann, sind die Röteln meldepflichtig. Man schätzt, dass weltweit etwa 100000 Neugeborene von einer Rötelnembryofetopathie (vor allem in Afrika, Südostasien und dem östlichen Mittelmeerraum) betroffen sind [33]. Das Rötelnvirus galt bis Mitte Oktober 2020 als das einzige Mitglied der Gattung Rubivirus, die wiederum zur Familie der Togaviridae gehört. Aus diesem Grund war der Ursprung der Röteln - im Gegensatz zu anderen als Zoonosen identifizierten Infektionskrankheiten - nicht bekannt, denn nur wenn andere Arten bekannt sind, kann man Verwandschaftsbeziehungen und damit auch evolutionäre Entwicklungen untersuchen. Unabhängig voneinander entdeckten 2 Forscherteams kürzlich 2 neue Virenarten aus der Gattung Rubivirus, was den zoonotischen Ursprung der Röteln sehr wahrscheinlich macht.

Der Letztautor der bemerkenswerten, im Fachblatt Nature erschienenen Publikation, Tony L. Goldberg, und sein ehemaliger Doktorand Andrew Bennett entdeckten eines der neuen Viren in anscheinend gesunden Rundblattnasen-Fledermäusen (Hipposideros cyclops), die in einem Nationalpark in Uganda gefangen wurden. Sie nannten es nach der Region Ruteete in Uganda und dem lokalen Wort für Fledermaus Ruhugu-Virus (RuhV). Das Genom des Virus ist sehr ähnlich mit dem des Röteln-Virus. Das Protein, das mit den Immunzellen des Wirts interagiert, war bei beiden Viren nahezu identisch. Während der Vorbereitungen der Publikation erfuhren die Wissenschaftler, dass eine Forschergruppe unter der Leitung von Martin Beer am Friedrich-Loeffler-Institut auf der Insel Riems bei Greifswald im Hirngewebe eines Esels, eines Kängurus und eines südamerikanischen Wasserschweins (Capybara, das mit einem Gewicht von bis zu $66 \mathrm{~kg}$ und einer Länge von bis zu $130 \mathrm{~cm}$ größte Nagetier der Welt), die alle im Zoo an einer Enzephalitis verstorben waren, das ebenfalls mit dem Röteln-Virus verwandte Rustrella-Virus (RusV; genannt nach einer Lagune in der Ostsee) entdeckt hatte. Sie fanden dieses Virus später auch bei wilden Gelbhals-Feldmäusen im Zoo (und nur bei diesen) bzw. im Umkreis von 10 Kilometern, ohne dass bei den Mäusen eine Enzephalitis vorlag. Dies deutet darauf hin, dass diese Mausart ein natürliches Reservoir darstellt, aus dem das Virus auf die Zootiere übergetreten war. Beim Vergleich ihrer Daten stellten die Wissenschaftler fest, dass RuhV und RusV miteinander verwandt sind, und beschlossen, ihre Ergebnisse gemeinsam zu veröffentlichen [46]. „Jetzt wissen wir, dass jede Krankheit in den Buchstaben des MMR-Impfstoffs einen zoonotischen Ursprung hat", wird der Seniorautor in einem im Fachblatt Science publizierten Bericht zitiert [47]. Angesichts des genetischen Abstands zwischen Röteln und dem Ruhugu- sowie dem Rostrela-Virus halten es die Forscher für unwahrscheinlich, dass das Röteln-Virus direkt von einem der beiden neu entdeckten Viren abstammt. Vielmehr könnte es weitere, noch nicht entdeckte Viren wie die beiden jetzt neu entdeckten geben, von denen das Röteln-Virus dann durch Sprung zum Menschen seinen Ausgang genommen hat. Die Arbeit unterstreicht damit die Bedeutung des One-Health-Ansatzes in der Medizin, der anerkennt, dass die Gesundheit von Menschen eng mit der von Tieren und der Umwelt verbunden ist. Von Bedeutung ist beispielsweise die Erkenntnis, dass das Rustrela-Virus offenbar in der Lage ist, sowohl Plazentaals auch Beuteltier-Säugetiere zu infizieren, also eine hohe Flexibilität besitzt, was das Hin- und Herspringen zwischen verschiedenen Arten anbelangt. Dies könnte die Wahrscheinlichkeit weiterer Pandemien durch Abkömmlinge von ähnlichen Viren wie RuhV und RusV, die noch gar nicht entdeckt sind, steigern.

\section{Was kann man tun?}

Zu den globalen Umweltproblemen Klimawandel (globale Erwärmung), Ozonloch, verminderte Artenvielfalt, Anstieg der Weltbevölkerung, demografischer Wandel (Überalterung), globale Umweltverschmutzung und Degeneration der Böden kommen noch die Begrenztheit und ungleiche Verteilung von Ressourcen (Bodenschätze, Energie, Trinkwasser), das globale Wettrüsten, der internationale ideologisch getriebene Terror und weltweit 79,5 Millionen Menschen (Stand: 2020) auf der Flucht [41]. Diese Häufung und Brisanz von globalen Problemen macht es schwer, Optimismus zu verbreiten. Den dürfen wir dennoch nicht verlieren, wenn wir mit den Problemen tatsächlich fertig werden wollen. Und dabei könnte der Gedanke von „einer Gesundheit“ - von Mensch, Tier und Erdball - helfen. Auf gesellschaftspolitischer Ebene ist mit den Institutionen, die neu entstanden sind oder sich intensiv mit diesem Gedanken befassen, sicherlich schon viel gewonnen. Sie müssen ausgebaut, noch besser vernetzt und dadurch gestärkt werden - als unabhängige Quellen von wirklichem Wissen, das dann in politische Entscheidungen eingehen kann.

Aber damit ist es nicht genug. Gerade die Corona-Krise zeigt, dass es auf das Verhalten jedes Einzelnen ankommt, wenn es darum geht, wie wir alle durch diese Krise kommen. Ein paar Beispiele hierfür seien aus der wissenschaftlichen Literatur kurz angeführt.

Nahrung: Eine schöne, im vergangenen Jahr in PNAS publizierte Arbeit geht der Frage nach, was wir - im Hinblick auf unsere Gesundheit und auf die Gesundheit des Planeten Erde - essen sollten [13]. Hierzu wurden 15 Nahrungsmittel sowohl auf ihre Mortalität und Morbidität als auch auf ihren ökologischen Fußabdruck hin miteinander verglichen. Das klingt zunächst ziemlich schwierig, denn wie sollte man das Risiko eines Dickdarmkrebses mit dem $\mathrm{CO}_{2}$-Ausstoß - jeweils ausgelöst durch eine zusätzliche Portion täglich von z. B. Olivenöl, Salami, Vollkornmüsli, Limonade, Käse, Obst oder Gemüse - vergleichen?

Die Lösung: Man bringt die 15 untersuchten Nahrungsmittel im Hinblick auf die gemessenen abhängigen Variablen - die unterschiedlicher gar nicht sein könnten - in eine Rangreihenfolge, von „Platz 1 “ bis „Platz 15“, und kann dann tatsächlich den Rang auf jeder Variable vergleichen. Ein Beispiel: Zur Produktion von einem Liter Limonade braucht man genau einen Liter Wasser, wohinge- 
gen man zur Produktion von einem Liter Olivenöl oder einem Kilogramm Nüssen Hunderte von Litern Wasser braucht. Olivenöl und Nüsse sind gesund, brauchen aber viel Wasser und sind daher ungesund für den Globus; Limonade hingegen braucht wenig Wasser, ist aber ungesund, weil sie sehr viel Zucker enthält. Glücklicherweise fällt die Auswahl nicht bei allen Nahrungsmitteln so schwer: Obst und Gemüse sind für den Menschen und die Erde gut; das Gegenteil ist bei Fleisch und Wurst der Fall ( $\triangleright$ Abb. $\mathbf{8})$.

Geld: Hat man erst einmal verstanden, dass die Funktion von Banken darin besteht, Geld von Leuten, die zu viel davon haben, denen zu geben, die keines haben, dafür aber Ideen, wie man Geld vermehren könnte. Man gibt es dann am liebsten denen, die am meisten Geld für's eingesetzte Geld rausholen, so schnell wie möglich. Daraus folgt, dass diejenigen, die einfach nur Ressourcen aus der Natur fördern, die schon da sind, bessere Chancen auf rasche und große Gewinne haben als diejenigen, die erst ein neues Produkt entwickeln, zur Marktreife bringen, produzieren und schließlich vermarkten möchten. Nach Öl oder Diamanten suchen, etwas finden, herausholen und verkaufen geht schneller. Auch Wald roden, etwas anbauen und dann verkaufen geht schneller. Hinzu kommt, dass die Industrie recht gut kontrolliert und reguliert arbeitet, Wald- und Minenarbeiter jedoch große Schäden anrichten können, was über lange Zeit unbeobachtet und unbemerkt bleibt. An unbewohnten Geisterstädten und unbelebten, weil durch Abraum vergifteten „Mondlandschaften“, wo früher unberührte Natur war, kann man erkennen, dass niemand für die Schäden aufkommt, wenn der Profit erst einmal gemacht ist. Die Ökonomie nennt solche „unerwünschten Wirkungen“ „negative Externalitäten“ die man eigentlich in den Preis eines Produktes mit einrechnen („internalisieren“) müsste, was jedoch meist nicht geschieht. Kernkraftwerke galten so lange als „Iukrativ“, wie man die Kosten der Endlagerung der radioaktiven Abfälle nicht in den Strompreis mit einrechnete. Wenn man also beim Investieren nur - und wirklich nur - auf das Geld (d. h. den Return of Investment) schaut, wird sich an dieser Sachlage nichts ändern. Wenn aber erst einmal klar ist, dass Schäden an Natur und Gesundheit langfristig teuer sind, und dass dieses Geld eigentlich vom Gewinn abzuziehen ist (wenn man negative Externalitäten internalisiert, steigt der Preis des Produkts, womit seine Konkurrenzfähigkeit und Vermarktbarkeit sinken dürfte sich herumsprechen, dass man Investitionen grundsätzlich anders bewerten sollte).

Es wird also Zeit, dass vernünftiger investiert wird - von jedem von uns. Wo beispielsweise steckt das Geld für Ihre Rente? Wurde es nachhaltig investiert? - Der WWF ${ }^{10}$ meint: Wenn ein jeder nachfragen würde, gäbe es vielleicht ein größeres Interesse der Investoren hier verantwortlicher zu handeln.

Unsere Reaktionen auf die jetzige Krise - die Corona-Pandemie - stimmen optimistisch: Wenn es wirklich sein muss, sind wir zu enormen Verhaltensänderungen innerhalb weniger WOCHEN in der Lage! Und dabei könnte der Gedanke von „einer Gesundheit“ - von Mensch, Tier und Erdball - helfen: zur Bewältigung der nächsten, wirklich großen Krise [44].

10 WWF: World Wildlife Fund For Nature. Vgl dessen Initiative: Make My Money Matter (https://makemymoneymatter.co.uk/; abgerufen am $5.11 .2020)$

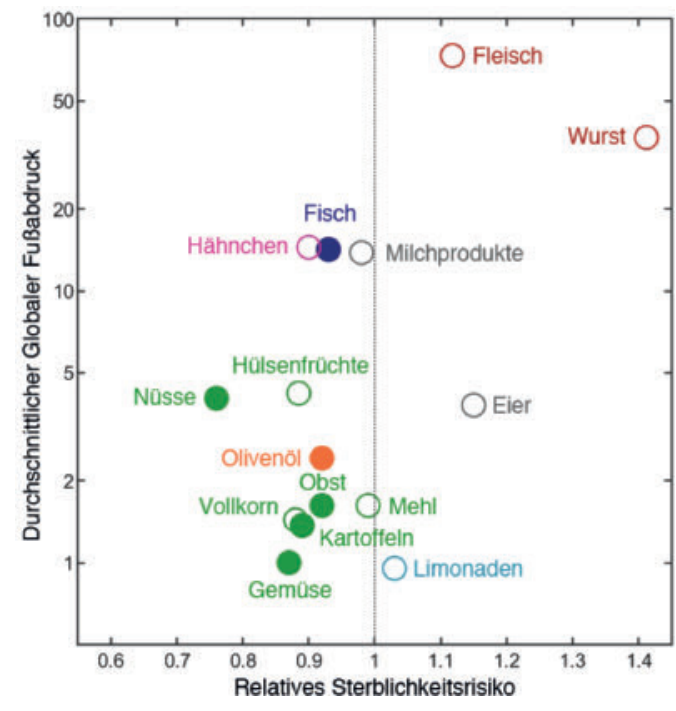

Abb. 8 Globaler Fußabdruck und Mortalität verschiedener Nahrungsmittel. Auf der $x$-Achse ist das relative Sterblichkeitsrisiko (>1: erhöht, <1: vermindert) und auf der $y$-Achse der ökologische Fußabdruck (gemessen anhand von 5 Variablen) im Verhältnis zur Produktion einer Portion Gemüse $(=1)$ logarithmisch aufgetragen. Die Angaben beziehen sich auf den Verzehr einer zusätzlichen täglichen Portion der folgenden Lebensmittelgruppen (gefüllte Kreise: signifikant von 1 abweichendes Mortalitätsrisiko, Kreis, keine signifikante Abweichung): Müsli (Körner, $30 \mathrm{~g}$ Trockengewicht); Müsli (verarbeitete Körner, $30 \mathrm{~g}$ Trockengewicht); Früchte (100 g); Gemüse (100 g); Nüsse (28 g); Hülsenfrüchte ( $50 \mathrm{~g}$ Trockengewicht); Kartoffeln (150 g); Fisch (100 g); Milchprodukte (200 g); Eier (50 g); Huhn (100 g); Fleisch (100 g); Wurst (50 g); Limonade (225g) und Olivenöl $(10 \mathrm{~g})$. Grün: nur minimal verarbeitete pflanzliche Lebensmittel; dunkelblau: Fisch; grau: Milchprodukte und Eier; rosa: Huhn; rot: Fleisch (Rind-, Lamm-, Ziegen- und Schweinefleisch) und Wurst; hellblau: Limonaden (mit Zucker gesüßte Getränke); Orange: Olivenöl (nach Daten aus [13]).

\section{Literatur}

[1] Dornelas M, Madin JS. Novel communities are a risky business. Science 2020; 370: 164-165

[2] Pandolfi JM, Staples TL, Kiessling W. Increased extinction in the emergence of novel ecological communities. Science 2020; 370: 220-222

[3] Vidal MC, Wang SP, Rivers DM, et al. Species richness and redundancy promote persistence of exploited mutualisms in yeast. Science 2020; 370: $346-350$

[4] Alvarez LW, Alvarez W, Asaro F, et al. Extraterrestrial cause for the Cretaceous/Tertiary extinction: Science 1980; 208: 1095-1108

[5] Antonelli A, Fry C, Smith R], et al. State of the World's Plants and Fungi 2020. Royal Botanic Gardens, Kew 2020; doi.org/10.34885/172; abgerufen am 31.10.2020

[6] Amuasi JH, Walzer C, Heymann D, et al. Calling for a COVID-19 One Health Research Coalition. Lancet 2020; 395: 1543-1544

[7] Ars Technica. The American Way of Life is Not Negotiable 2020 https:// arstechnica.com/civis/viewtopic.php?f=24\&t=981983; abgerufen am 26.10.2020

[8] Bestion E, Teyssier A, Richard M, et al. Live Fast, Die Young: Experimental Evidence of Population Extinction Risk due to Climate Change. PLoS Biol 2015; 13(10): e1002281 
[9] Budd L, Morag Bell, Brown T. Of plagues, planes and politics: Controlling the global spread of infectious diseases by air. Polit Geogr 2009; 28: 426-435

[10] Butler CD. Infectious disease emergence and global change: thinking systemically in a shrinking world. Infect Dis Poverty 2012; 1: 5

[11] Centers for Disease Control and Prevention, CDC 2019 One Health Zoonotic Disease Prioritization Process Overview . https://www.cdc. gov/onehealth/what-we-do/zoonotic-disease-prioritization/fact-sheet. html). www.cdc.gov; abgerufen am 9.10.2020

[12] Christidis P, Christodoulou A. The Predictive Capacity of Air Travel Patterns During the Global Spread of the COVID-19 Pandemic: Risk, Uncertainty and Randomness. Int J Environ Res Public Health 2020; 17: 3356

[13] Clark MA, Springmann M, Hill J, et al. Multiple health and environmental impacts of foods. PNAS 2019; 116: 23357-23362

[14] Crutzen PJ, Stoermer EF. The Anthropocene. The International Geosphere-Biosphere Programme, IGBP; Newsletter 2000; 41: 17-18

[15] Crutzen PJ. Geology of mankind. Nature 2002; 415: 23

[16] Deem SL, Lane-deGraaf KE, Elizabeth A et al Introduction to One Health: An Interdisciplinary Approach to Planetary Health. New York: Wiley-Blackwell; 2018

[17] FSIN, Food Security Information Network. Global Report on Food Crises. Joint analysis for better decisions 2020 https://www.fsinplatform. org/; abgerufen am 23.10.2020

[18] Global Footprint Network. Past Earth Overshoot Days. In: Earth Overshoot Day 2020 https://www.overshootday.org/newsroom/past-earthovershoot-days/abgerufen am 23.10.2020

[19] Greenpeace. Internationale Klimakonferenzen 2020 https://www. greenpeace.de/themen/klimawandel/klimaschutz/internationaleklimakonferenzen; abgerufen am 26.10.2020

[20] Hatcher M], Dick JTA, Dunn AM. Disease emergence and invasions. Funct Ecol 2012; 26: 1275-1287

[21] Henehan M], Ridgwell A, Thomas E, et al. Rapid ocean acidification and protracted Earth system recovery followed the end-Cretaceous Chicxulub impact. PNAS 2019; 116: 22500-22504

[22] Hildebrand AR, Penfield GT, Kring DA, et al. Chicxulub Crater: A possible Cretaceous/Tertiary boundary impact crater on the Yucatán Peninsula, Mexico. Geology 1991; 19: 867-871

[23] Hillienhof A. One-Health-Ansatz: WHO stellt Leitfaden zur Bekämpfung von Zoonosen vor. Deutsches Ärzteblatt 209; 116 (14): A-706

[24] IBN, Institut für Biodiversität - Netzwerk. Intergovernmental Platform on Biodiversity and Ecosystem Services (IPBES). Institut für Biodiversität, Regensburg 2020 https://biodiv.de/biodiversitaet-infos/ipbes. html; abgerufen am 1.11.2020

[25] IPBES, Intergovernmental Science-Policy Platform on Biodiversity and Ecosystem Services. IPBES Workshop on Biodiversity and Pandemics. Report vom 29.10.2020 https://ipbes.net/sites/default/ files/2020-10/20201028\%20IPBES\%20Pandemics\%20Workshop\%20Report\%20Plain\%20Text\%20Final_0.pdf; abgerufen am 31.10.2020 und Executive Summary vom 29.10.2020 https://ipbes.net/sites/default/ files/2020-10/IPBES\%20Pandemics\%20Workshop\%20Report\%20Executive\%20Summary\%20Final.pdf; abgerufen am 31.10.2020

[26] IUCN, International Union for Conservation of Nature. Summary statistics 2020. www.iucnredlist.org/resources/summary-statistics, Table 1b; abgerufen am 3.11.2020

[27] Jack JC, Gonet J, Mease A, et al. Traditional Knowledge underlies One Health. Science 2020; 369: 1576
[28] MacKenzie D. What price for food. New Scientist 2008; 2660 , 14.6.2008. www.newscientist.com/article/mg19826601-600-whatprice-more-food/; abgerufen am 7.5.2019

[29] Maganga GD, Pinto A, Mombo IM, et al. Genetic diversity and ecology of coronaviruses hosted by cave-dwelling bats in Gabon. Sci Rep 2020; 10: 7314

[30] Marshall M. Controlling deforestation and wildlife trade could prevent pandemics. New Scientist 2020; 29.10.2020 https://www.newscientist.com/article/2258527-controlling-deforestation-and-wildlife-tradecould-prevent-pandemics/\#ixzz6cToJEaNU; abgerufen am 29.10.2020

[31] O'Bryan C], Garnett ST, Fa JE, et al. The importance of indigenous peoples' lands for the conservation of terrestrial mammals. Conservation Biology 2020; https://doi.org/10.1111/cobi.13620

[32] One Health Initiative Task Force, OHITF. One Health: A New Professional Imperative. Final Report 2008. www.avma.org/sites/default/files/ resources/onehealth_final.pdf; abgerufen am 9.10.2020

[33] Robertson SE, Featherstone DA, Gacic-Dobo M, et al. Rubella and congenital rubella syndrome: global update. Rev Panam Salud Publica/ Pan Am J Public Health 2008; 14: 306-315

[34] Roser M, Ritchie H. Food Prices. OurWorldInData.org 2013 https:// ourworldindata.org/food-prices; abgerufen am 30.10.2020

[35] Schulte P, Alegret L, Arenillas I, et al. The Chicxulub asteroid impact and mass extinction at the Cretaceous-Paleogene boundary. Science 2010; 327: 1214-1218

[36] Spitzer M. Demographie, Dynamik und Demokratie. Nervenheilkunde 2013; 32: 778-783

[37] Steffen W, Crutzen PJ, McNeill JR. The Anthropocene: Are Humans Now Overwhelming the Great Forces of Nature. AMBIO 2007; 36: 614-621

[38] Streck R. Demokratie- oder Hungerrevolten? Telepolis, Heise online, 21.2.2011. www.heise.de/tp/features/Demokratie-oder-Hungerrevolten-3388737.html; abgerufen am 21.10.2020

[39] Tatem AJ, Hay SI, Rogers DJ. Global traffic and disease vector dispersal. PNAS 2006; 103: 6242-6247

[40] United Nations Environment Programme, UNEP. Global Biodiversity Assessment. Heywood VH (Hrsg). Cambridge: University Press; 1995

[41] UNO Flüchtlingshilfe. Zahlen und Fakten zu Menschen auf der Flucht 2020. www.uno-fluechtlingshilfe.de/informieren/fluechtlingszahlen/

[42] Urban MC. Accelerating extinction risk from climate change. Science 2015; 348: 571-573

[43] Urdal HA. Clash of generations? Youth bulges and political violence. United Nations Department of Economic and Social Affairs, Population Division. Expert Paper 2012/1. www.un.org/en/development/desa/ population/publications/pdf/expert/2012-1_Urdal_Expert-Paper.pdf; abgerufen am 4.9.2013

[44] Vaughan A. How the coronavirus has impacted climate change - for good and bad Global warming has become a forgotten crisis during the coronavirus pandemic. New Scientist 2020; 14.10.2020 www. newscientist.com/article/mg24833040-900-how-the-coronavirushas-impacted-climate-change-for-good-and-bad/; abgerufen am 5.11.2020

[45] Wake DB, Vredenburg VT. Colloquium paper: are we in the midst of the sixth mass extinction? A view from the world of amphibians. PNAS 2008; 105 (Suppl 1): 11466-11473

[46] Bennett AJ, Adrian C. Paskey AC, et al. Relatives of rubella virus in diverse mammals. Nature 2020; 586: 424-42

[47] Gibbons A. Newly found viruses suggest rubella originated in animals. Science 2020; 370: 15 